PROCEEDINGS OF THE

AMERICAN MATHEMATICAL SOCIETY

Volume 131, Number 7, Pages 2189-2199

S 0002-9939(02)06851-X

Article electronically published on November 13, 2002

\title{
HECKE ALGEBRAS OF SEMIDIRECT PRODUCTS
}

\author{
MARCELO LACA AND NADIA S. LARSEN \\ (Communicated by David R. Larson)
}

\begin{abstract}
We consider group-subgroup pairs in which the group is a semidirect product and the subgroup is contained in the normal part. We give conditions for the pair to be a Hecke pair and we show that the enveloping Hecke algebra and Hecke $C^{*}$-algebra are canonically isomorphic to semigroup crossed products, generalizing earlier results of Arledge, Laca and Raeburn and of Brenken.
\end{abstract}

\section{INTRODUCTION}

A group-subgroup pair $\left(\Gamma, \Gamma_{0}\right)$ is called a Hecke pair if each double coset $\Gamma_{0} \gamma \Gamma_{0}$ is a finite union of left cosets $\gamma_{i} \Gamma_{0}$. By considering inverses, one sees that it is equivalent to require that each double coset is the union of finitely many right cosets. This situation is variously referred to by saying that $\Gamma_{0}$ is a Hecke subgroup or an almost normal subgroup of $\Gamma$. See [7] for more details.

To each Hecke pair one associates a Hecke ${ }^{*}$-algebra $\mathcal{H}\left(\Gamma, \Gamma_{0}\right)$, by endowing the set of $\Gamma_{0}$-bi-invariant complex valued functions on $\Gamma$ that are supported in finitely many double cosets with a convolution product defined by the (finite) sum

$$
f * g(\gamma)=\sum_{\gamma_{1} \in \Gamma_{0} \backslash \Gamma} f\left(\gamma \gamma_{1}^{-1}\right) g\left(\gamma_{1}\right),
$$

and an involution defined by $f^{*}(\gamma)=\overline{f\left(\gamma^{-1}\right)}$. The characteristic function of $\Gamma_{0}$ is the identity element. Hecke algebras have been around for quite some time but their $\mathrm{C}^{*}$-algebraic version has only recently begun to get some attention, initially in the work of Binder [2] and Bost and Connes [4, and more recently in that of Hall [6] and Tzanev [16]. One defines the reduced Hecke $C^{*}$-algebra $C_{r}^{*}\left(\Gamma, \Gamma_{0}\right)$ to be the closure of $\mathcal{H}\left(\Gamma, \Gamma_{0}\right)$ in the natural representation on $\ell^{2}\left(\Gamma_{0} \backslash \Gamma\right)$ obtained using the above convolution formula with $g \in \ell^{2}\left(\Gamma_{0} \backslash \Gamma\right)$. A universal version $C_{u}^{*}\left(\Gamma, \Gamma_{0}\right)$, through which all representations of $\mathcal{H}\left(\Gamma, \Gamma_{0}\right)$ on Hilbert space factor, is not so readily obtained because in some cases there are generators that do not have uniformly bounded norms in all *-representations; see [6, Example 2.3.3]. To some extent this difficulty can be sidestepped by restricting the attention to representations that do not increase a certain Banach algebra norm on $\mathcal{H}\left(\Gamma, \Gamma_{0}\right)$; see [16].

Received by the editors July 15, 2001 and, in revised form, January 22, 2002 and March 5, 2002 .

2000 Mathematics Subject Classification. Primary 46L55.

The first author was supported by the Deutsche Forschungsgemeinschaft [SFB 478].

The second author was supported by the Danish Natural Science Research Council and The Carlsberg Foundation. 
The present work is motivated by the realization of various Hecke $\mathrm{C}^{*}$-algebras as semigroup crossed products. Following [9, 1], but especially guided by [5], we are first led to consider the semigroup of elements of $\Gamma$ whose left coset is right invariant, and hence a double coset. We observe that if this semigroup generates all of $\Gamma$ by left quotients, then the problem with unbounded norms does not arise because $\mathcal{H}\left(\Gamma, \Gamma_{0}\right)$ has a generating set consisting of isometries, providing a simple proof of a result of Hall concerning the existence of the universal Hecke $\mathrm{C}^{*}$-algebra [6. Corollary 4.6].

Our main results are about pairs consisting of a semidirect product group $\Gamma=$ $N \rtimes G$ and a subgroup $\Gamma_{0} \subset N$. We give sufficient conditions on the action of $G$ on $N$ for $\left(\Gamma, \Gamma_{0}\right)$ to be a Hecke pair, and we realize the Hecke algebra, and $\mathrm{C}^{*}$-algebra, as semigroup crossed products. These results unify and generalize previous realizations of Hecke $\mathrm{C}^{*}$-algebras as semigroup crossed products obtained in 9 , 1, 5, 13.

Our approach relies freely on the basic results from Section 1 of [5] where the generality is appropriate for our purposes, but the techniques used to obtain our main results are independent of the rest of [5. Indeed, we are able to give simpler proofs of more general statements. Notably, by considering a different set of generating monomials, inspired by those of [1], we have done away with the quasi-lattice structure on $G$. Perhaps more significantly, we have removed the assumption that $\Gamma_{0}$ be normal in $N$; instead we assume that $\left(N, \Gamma_{0}\right)$ is a Hecke pair, and replace the group algebra $\mathbb{C}\left(N / \Gamma_{0}\right)$ with the Hecke algebra $\mathcal{H}\left(N, \Gamma_{0}\right)$.

We would like to thank Iain Raeburn and George Willis for several helpful conversations.

\section{Hecke algebras of SEMidirect PRODUCtS}

Definition 1.1 (cf. 4]). For each $\gamma \in \Gamma$, let $R(\gamma)$ (respectively, $L(\gamma)$ ) be the cardinality of the image of $\Gamma_{0} \gamma \Gamma_{0}$ in $\Gamma_{0} \backslash \Gamma$ (respectively, in $\Gamma / \Gamma_{0}$ ). It is clear that $L(\gamma)=R\left(\gamma^{-1}\right)$.

Notation. Throughout we denote by $[\gamma]$ the characteristic function of the double coset of an element $\gamma \in \Gamma$.

Remark 1.2. The Hecke algebra $\mathcal{H}\left(\Gamma, \Gamma_{0}\right)$ is the linear span of $\{[\gamma]: \gamma \in \Gamma\}$. The product of two elements of this spanning set is given by

$$
([\gamma][\delta])(x)=\left|\Gamma_{0} \backslash\left(\Gamma_{0} \gamma^{-1} \Gamma_{0} x \cap \Gamma_{0} \delta \Gamma_{0}\right)\right|,
$$

from which one easily sees that the support of $[\gamma][\delta]$ is $\Gamma_{0} \gamma \Gamma_{0} \delta \Gamma_{0}$.

From Theorems 1.2 and 1.4 of [5] we know that the set

$$
\Sigma:=\{\sigma \in \Gamma: L(\sigma)=1\}
$$

is a semigroup on which $R$ is multiplicative, and that the map

$$
\sigma \in \Sigma \mapsto W_{\sigma}:=R(\sigma)^{-1 / 2}[\gamma] \in \mathcal{H}\left(\Gamma, \Gamma_{0}\right)
$$

is a representation of $\Sigma$ by isometries in $\mathcal{H}\left(\Gamma, \Gamma_{0}\right)$. We will need the following supplement of this.

Lemma 1.3. For every $\gamma \in \Gamma$ and $\sigma \in \Sigma$,

(i) the product $[\gamma][\sigma]$ is a positive integer multiple of $[\gamma \sigma]$, and $L(\gamma \sigma)=L(\gamma)$; 
(ii) the product $[\sigma]^{*}[\gamma]$ is a positive integer multiple of $\left[\sigma^{-1} \gamma\right]$, and $R\left(\sigma^{-1} \gamma\right)=$ $R(\gamma)$.

Proof. Clearly $\sigma \in \Sigma$ if and only if $\Gamma_{0} \sigma \Gamma_{0}=\sigma \Gamma_{0}$, from which it follows that $\Gamma_{0} \gamma \sigma \Gamma_{0}=\Gamma_{0} \gamma \Gamma_{0} \sigma \Gamma_{0}=\left(\bigsqcup_{i=1}^{L(\gamma)} \gamma_{i} \Gamma_{0}\right) \sigma \Gamma_{0}=\bigsqcup_{i=1}^{L(\gamma)} \gamma_{i} \sigma \Gamma_{0}$. Since the support of $[\gamma][\sigma]$ is $\Gamma_{0} \gamma \Gamma_{0} \sigma \Gamma_{0}$, we obtain (i), from which (ii) follows on considering adjoints and replacing $\gamma$ by $\gamma^{-1}$.

Proposition 1.4. Suppose $\left(\Gamma, \Gamma_{0}\right)$ is a Hecke pair such that $\Sigma^{-1} \Sigma=\Gamma$. Then $W_{\beta}^{*} W_{\alpha}=W_{\tau}^{*} W_{\sigma}$ whenever $\beta^{-1} \alpha=\tau^{-1} \sigma$ and the set $\left\{W_{\tau}^{*} W_{\sigma}: \tau, \sigma \in \Sigma\right\}$ is a linear basis of $\mathcal{H}\left(\Gamma, \Gamma_{0}\right)$. Moreover, the universal $C^{*}$-enveloping algebra $C_{u}^{*}\left(\Gamma, \Gamma_{0}\right)$ of $\mathcal{H}\left(\Gamma, \Gamma_{0}\right)$ is a quotient of the universal $C^{*}$-algebra of isometric representations of $\Sigma$.

Remark 1.5. Since the semigroup $\Sigma$ is cancellative, because it is contained in $\Gamma$, by assuming that $\Sigma^{-1} \Sigma=\Gamma$ (or equivalently that $\Sigma \gamma \cap \Sigma \gamma^{\prime} \neq \emptyset$ for every pair $\gamma, \gamma^{\prime} \in \Gamma$ ) we are saying that $\Sigma$ is an Ore semigroup with $\Gamma$ its group of left quotients. The proposition asserts in particular that $C_{u}^{*}\left(\Gamma, \Gamma_{0}\right)$ exists, proved in [6. Corollary 4.6]; see also [5 Proposition 2.8].

Proof. Let $\tau, \sigma \in \Sigma$; from the lemma we know that

$$
[\tau]^{*}[\sigma]=K_{\sigma, \tau}\left[\tau^{-1} \sigma\right]
$$

where $K_{\sigma, \tau}$ is a positive integer. Hence, up to positive constants, the products $W_{\tau}^{*} W_{\sigma}$ are the characteristic functions of all the double cosets, so their linear span is $\mathcal{H}\left(\Gamma, \Gamma_{0}\right)$.

From this it already follows that $\mathcal{H}\left(\Gamma, \Gamma_{0}\right)$ is generated by isometries and that the universal $\mathrm{C}^{*}$-enveloping algebra for $\mathcal{H}\left(\Gamma, \Gamma_{0}\right)$ exists and is a quotient of the universal $\mathrm{C}^{*}$-algebra of isometric representations of $\Sigma$. The key here is, of course, that isometries have norm 1 in any nontrivial Hilbert space representation, so the basic conditions for the existence of the universal $\mathrm{C}^{*}$-algebra (see e.g. [3]) are satisfied.

Since the characteristic functions of distinct double cosets are disjoint, to conclude that the given set is a linear basis of $\mathcal{H}\left(\Gamma, \Gamma_{0}\right)$ all we have to prove is that $W_{\beta}^{*} W_{\alpha}=W_{\tau}^{*} W_{\sigma}$ whenever $\beta^{-1} \alpha=\tau^{-1} \sigma$, with $\alpha, \beta, \sigma$ and $\tau$ in $\Sigma$. For this it suffices to show that the expression

$$
\frac{K_{\sigma, \tau}}{R(\tau)^{1 / 2} R(\sigma)^{1 / 2}}
$$

depends only on the left quotient $\tau^{-1} \sigma$. Since $\Sigma$ is directed, that is, $\Sigma \gamma \cap \Sigma \gamma^{\prime} \neq \emptyset$, it is enough to show that for each $\gamma \in \Sigma$ the value of the above expression does not change when we replace $\sigma$ and $\tau$ by $\gamma \sigma$ and $\gamma \tau$, respectively, for which it suffices to show that $K_{\gamma \sigma, \gamma \tau}=R(\gamma) K_{\sigma, \tau}$ because $R$ is multiplicative on $\Sigma$. In order to prove this we first compute $K_{\sigma, \tau}$ by evaluating the convolution product at $\tau^{-1} \sigma$ using Remark 1.2

$$
\begin{aligned}
K_{\sigma, \tau} & =\left(\left[\tau^{-1}\right][\sigma]\right)\left(\tau^{-1} \sigma\right) \\
& =\left|\Gamma_{0} \backslash\left(\Gamma_{0} \tau \Gamma_{0} \tau^{-1} \sigma \cap \Gamma_{0} \sigma \Gamma_{0}\right)\right| \\
& =\left|\Gamma_{0} \backslash\left(\tau \Gamma_{0} \tau^{-1} \sigma \cap \sigma \Gamma_{0}\right)\right| \\
& =\left|\Gamma_{0} \backslash\left(\tau \Gamma_{0} \tau^{-1} \cap \sigma \Gamma_{0} \sigma^{-1}\right)\right| .
\end{aligned}
$$


The claim then follows by applying the basic fact $|A \backslash C|=|A \backslash B||B \backslash C|$, valid for every group inclusion $A \subset B \subset C$, to the inclusions

$$
\Gamma_{0} \subset \gamma \Gamma_{0} \gamma^{-1} \subset \gamma \tau \Gamma_{0} \tau^{-1} \gamma^{-1} \cap \gamma \sigma \Gamma_{0} \sigma^{-1} \gamma^{-1},
$$

and observing that $R(\gamma)=\left|\Gamma_{0} \backslash\left(\gamma \Gamma_{0} \gamma^{-1}\right)\right|$ and that

$$
\left|\left(\gamma \Gamma_{0} \gamma^{-1}\right) \backslash\left(\gamma \tau \Gamma_{0} \tau^{-1} \gamma^{-1} \cap \gamma \sigma \Gamma_{0} \sigma^{-1} \gamma^{-1}\right)\right|=\left|\Gamma_{0} \backslash\left(\tau \Gamma_{0} \tau^{-1} \cap \sigma \Gamma_{0} \sigma^{-1}\right)\right| .
$$

We pause to recall that when $\alpha$ is an action of a semigroup $S$ by endomorphisms of a unital $\mathrm{C}^{*}$-algebra $A$, one defines a covariant representation to be a pair $(\pi, V)$ in which $\pi$ is a unital representation of $A$ and $V$ is a representation of $S$ by isometries such that $\pi\left(\alpha_{s}(a)\right)=V_{s} \pi(a) V_{s}^{*}$ for every $a \in A$ and $s \in S$. The associated semigroup crossed product is the $\mathrm{C}^{*}$-algebra generated by a universal covariant representation, which, by definition, is one through which every covariant representation factors; see [8, 15] for more details. The analogous concept in the category of *-algebras is defined in the obvious way and we refer to it as the *-algebraic semigroup crossed product.

Remark 1.6. Since $W$ is a semigroup of isometries, there is a semigroup of endomorphisms (of $\mathcal{H}\left(\Gamma, \Gamma_{0}\right)$ and of $C_{u}^{*}\left(\Gamma, \Gamma_{0}\right)$ ) defined by $\alpha_{s}(b):=W_{s} b W_{s}^{*}$. Let $B$ denote the ${ }^{*}$-subalgebra of $\mathcal{H}\left(\Gamma, \Gamma_{0}\right)$ generated by the projections $W_{s} W_{s}^{*}$ with $s \in \Sigma$, in other words, $B$ is the linear span of the products of such projections. Since $W_{\sigma}\left(\prod_{j} W_{s_{j}} W_{s_{j}}^{*}\right) W_{\sigma}^{*}=\prod_{j} W_{\sigma s_{j}} W_{\sigma s_{j}}^{*}$, the endomorphisms leave $B$ invariant, indeed, $B$ is the smallest invariant unital *-subalgebra. It is easy to show that the associated semigroup crossed product $B \rtimes \Sigma$ maps onto the Hecke algebra in a canonical way. However, there is in principle no reason why this map should be injective in general, because the covariance relation (which takes place in the algebra $B$ ) might not capture all the relations satisfied by the generators of the Hecke algebra. By the same token, if $N$ is a normal subgroup of $\Gamma$ and $\Gamma_{0}$ is normal in $N$, there is a surjective homomorphism of $\mathbb{C}\left(N / \Gamma_{0}\right) \rtimes \Sigma$ onto $\mathcal{H}\left(\Gamma, \Gamma_{0}\right)$. This shows that the conclusion of [5] Proposition 2.10] holds without any assumptions on least upper bounds.

When $\Gamma$ is a semidirect product we will be able to say more. Recall that if the group $G$ acts on the group $N$ by automorphisms $\psi_{g}$, one defines the semidirect product group $\Gamma=N \rtimes G$ by endowing the set $N \times G$ with the twisted product $(n, g)(m, h)=\left(n \psi_{g}(m), g h\right)$. One can then view $G$ and $N$ as subgroups of $\Gamma$, with $N$ normal, and write the automorphisms $\psi_{g}$ as inner automorphisms obtained by conjugation by elements of $G$, namely, $\psi_{g}(m)=g m g^{-1}$.

Proposition 1.7. Let $\Gamma:=N \rtimes G$ be a semidirect product group and let $\Gamma_{0}$ be a subgroup of $N$. Suppose that $S$ is a subsemigroup of $G$ such that $S^{-1} S=G$ and $s^{-1} \Gamma_{0} s \subset \Gamma_{0}$ for every $s \in S$. Then $\left(\Gamma, \Gamma_{0}\right)$ is a Hecke pair if and only if $\left(N, \Gamma_{0}\right)$ is a Hecke pair and $\left|s^{-1} \Gamma_{0} s \backslash \Gamma_{0}\right|<\infty$ for every $s \in S$. Furthermore, $R(s)=\left|s^{-1} \Gamma_{0} s \backslash \Gamma_{0}\right|$ for every $s \in S$, and the identity map gives an embedding $\mathcal{H}\left(N, \Gamma_{0}\right) \hookrightarrow \mathcal{H}\left(\Gamma, \Gamma_{0}\right)$.

Proof. The assumption $s^{-1} \Gamma_{0} s \subset \Gamma_{0}$ says that the action of $S^{-1}$ leaves $\Gamma_{0}$ invariant. This is also equivalent to saying that $S \subset \Sigma$, because $s^{-1} \Gamma_{0} s \subset \Gamma_{0}$ implies $\Gamma_{0} s \Gamma_{0}=$ $s \Gamma_{0}$. 
Assume first that $\Gamma_{0}$ is a Hecke subgroup of $\Gamma$. Then it is clearly also a Hecke subgroup of $N$, and for each $s \in S$ one has $\left|s^{-1} \Gamma_{0} s \backslash \Gamma_{0}\right|=\left|\Gamma_{0} \backslash s \Gamma_{0} s^{-1}\right|=\left|\Gamma_{0} \backslash \Gamma_{0} s \Gamma_{0} s^{-1}\right|$ $=\left|\Gamma_{0} \backslash\left(\Gamma_{0} s \Gamma_{0}\right)\right|=R(s)<\infty$.

Assume now that $\Gamma_{0}$ is a Hecke subgroup of $N$ and that $\left|s^{-1} \Gamma_{0} s \backslash \Gamma_{0}\right|<\infty$ for every $s \in S$. Let $\gamma \in \Gamma$ and write $\gamma=t^{-1} n s$ with $t, s \in S$ and $n \in N$ (an easy argument shows this is always possible because $S^{-1} S=G$ ). In order to show that $\left|\Gamma_{0} \backslash \Gamma_{0} t^{-1} n s \Gamma_{0}\right|$ is finite, we write

$$
\begin{array}{rlrl}
\Gamma_{0} t^{-1} n s \Gamma_{0} & =\Gamma_{0} t^{-1} \Gamma_{0} n \Gamma_{0} s \Gamma_{0} & \\
& =\bigcup_{j} \Gamma_{0} t^{-1} \Gamma_{0} n \Gamma_{0} \gamma_{j} \quad & & \text { because } s \Gamma_{0}=\Gamma_{0} s \Gamma_{0}=\bigsqcup_{j=1}^{R(s)} \Gamma_{0} \gamma_{j} \\
& =\bigcup_{j} \Gamma_{i} t^{-1} \Gamma_{0} n_{i} \gamma_{j} \quad \text { because } \Gamma_{0} n \Gamma_{0}=\bigsqcup_{i=1}^{R(n)} \Gamma_{0} n_{i} \\
& =\bigcup_{j} \bigcup_{i} \Gamma_{0} t^{-1} n_{i} \gamma_{j},
\end{array}
$$

which is a union of at most $R(s) R(n)$ right cosets. This proves that $\left(\Gamma, \Gamma_{0}\right)$ is a Hecke pair, and hence that $R(s)=\left|s^{-1} \Gamma_{0} s \backslash \Gamma_{0}\right|$, as shown above. Finally, the operations defined on $\mathcal{H}\left(\Gamma, \Gamma_{0}\right)$ restrict to those on $\mathcal{H}\left(N, \Gamma_{0}\right)$, so the identity map gives a unital embedding of $*_{\text {-algebras }} \mathcal{H}\left(N, \Gamma_{0}\right) \hookrightarrow \mathcal{H}\left(\Gamma, \Gamma_{0}\right)$.

Remark 1.8. The set $S_{0}:=\left\{s \in G:\left|s^{-1} \Gamma_{0} s \backslash \Gamma_{0}\right|<\infty\right\}$ is itself always a semigroup, in fact, the largest possible subsemigroup of $G$ that can satisfy the conditions of hypothesis. If $S_{0}^{-1} S_{0}=G$, the proposition certainly applies with $S=S_{0}$, but in some situations one might be better off with a proper subsemigroup $S$ of $S_{0}$ that happens to generate $G$ by left quotients, too.

Theorem 1.9. Let $\Gamma:=N \rtimes G$ be a semidirect product group and let $\Gamma_{0}$ be a subgroup of $N$. Suppose that $S$ is a subsemigroup of $G$ such that $S^{-1} S=G$ and $\left|s^{-1} \Gamma_{0} s \backslash \Gamma_{0}\right|<\infty$ for every $s \in S$. If $\left(N, \Gamma_{0}\right)$ is a Hecke pair, then $\left(\Gamma, \Gamma_{0}\right)$ is a Hecke pair, with $R(s)=\left|s^{-1} \Gamma_{0} s \backslash \Gamma_{0}\right|$, and

(i) the elements of $\mathcal{H}\left(\Gamma, \Gamma_{0}\right)$ given by $\mu_{s}:=R(s)^{-1 / 2}[s]$ for $s \in S$ and $e(x):=$ $[x]$ for $x \in N$ satisfy the relations:

$\left(\mathfrak{h}_{1}\right) \mu_{s}^{*} \mu_{s}=I$ and $\mu_{s} \mu_{t}=\mu_{s t}$, for $s, t \in S$;

$\left(\mathfrak{h}_{2}\right) e(1)=I, e(x)^{*}=e\left(x^{-1}\right)$, and $e(x) e(y)=[x][y]$ (as in $\left.\mathcal{H}\left(N, \Gamma_{0}\right)\right)$; and

$\left(\mathfrak{h}_{3}\right) \mu_{s} e(x) \mu_{s}^{*}=R(s)^{-1} \sum_{\left\{[y]:\left[s^{-1} y s\right]=[x]\right\}} e(y)$, for $s \in S$ and $x \in N$;

and the set $\left\{\mu_{t}^{*} e(x) \mu_{s}: s, t \in S\right.$ and $\left.x \in N\right\}$ is a linear basis of $\mathcal{H}\left(\Gamma, \Gamma_{0}\right)$;

(ii) there is an action $\alpha$ of $S$ by injective corner endomorphisms of the Hecke *-algebra $\mathcal{H}\left(N, \Gamma_{0}\right)$ defined by

$$
\alpha_{s}(e(x)):=R(s)^{-1} \sum_{\left\{[y]:\left[s^{-1} y s\right]=[x]\right\}} e(y),
$$

and the Hecke ${ }^{*}$-algebra $\mathcal{H}\left(\Gamma, \Gamma_{0}\right)$ is canonically isomorphic to the ${ }^{*}$-algebraic semigroup crossed product $\mathcal{H}\left(N, \Gamma_{0}\right) \rtimes_{\alpha} S$, and to the universal unital *algebra with presentation $\left(\mathfrak{h}_{1}, \mathfrak{h}_{2}, \mathfrak{h}_{3}\right)$.

We point out that the given labelling of the elements of the linear basis of part (i) is not one-to-one, and Lemma 1.10 below deals precisely with this redundancy. 
Proof. The first assertion is from Proposition 1.7. A somewhat tedious but straightforward computation of convolution products in $\mathcal{H}\left(\Gamma, \Gamma_{0}\right)$ shows that the $\mu_{s}$ and $e(x)$ satisfy the relations $\left(\mathfrak{h}_{1}, \mathfrak{h}_{2}, \mathfrak{h}_{3}\right)$. Notice that relation $\left(\mathfrak{h}_{1}\right)$ simply says that $\mu$ is an isometric representation of $S$ (see Theorem 1.4 of [5]) and relation $\left(\mathfrak{h}_{2}\right)$ is just a restatement of the embedding of $\mathcal{H}\left(N, \Gamma_{0}\right)$ in $\mathcal{H}\left(\Gamma, \Gamma_{0}\right)$. In order to see that the set $\left\{\mu_{t}^{*} e(x) \mu_{s}: s, t \in S\right.$ and $\left.x \in N\right\}$ is a linear basis of $\mathcal{H}\left(\Gamma, \Gamma_{0}\right)$, recall that $\mu_{t}^{*} e(x) \mu_{s}$ is a function on $\Gamma$ and notice that its support is the set $\Gamma_{0} t^{-1} \Gamma_{0} x \Gamma_{0} s \Gamma_{0}=\Gamma_{0} t^{-1} x s \Gamma_{0}$. Since $\Gamma=S^{-1} N S$, all double cosets arise as supports. Thus the products $\mu_{t}^{*} e(x) \mu_{s}$ linearly span $\mathcal{H}\left(\Gamma, \Gamma_{0}\right)$, and we only have to prove that $\left\{\mu_{t}^{*} e(x) \mu_{s}: s, t \in S\right.$ and $\left.x \in N\right\}$ is a linearly independent set. Different supports are disjoint, so to prove linear independence it suffices to show that if two such products have the same support, then they coincide, which we do in the following lemma.

Lemma 1.10. For $t, s, \tau, \sigma \in S$ and $m, n \in N$, we have that $\left[\tau^{-1} m \sigma\right]=\left[t^{-1} n s\right]$ if and only if $\mu_{\tau}^{*}[m] \mu_{\sigma}=\mu_{t}^{*}[n] \mu_{s}$.

Proof. The 'if' part is immediate on considering supports. To prove the 'only if' part, begin by taking quotients modulo $N$ in $\left[\tau^{-1} m \sigma\right]=\left[t^{-1} n s\right]$ to see that $\tau^{-1} \sigma=t^{-1} s$. Since $S$ is directed there exist $r$ and $\gamma$ in $S$ such that $r t=\gamma \tau$ and $r s=\gamma \sigma$. Since $S \subset \Sigma$, Lemma 1.3 implies that the function $R$ is invariant under multiplication by elements of $S^{-1}$ on the left, so

$$
R(\sigma)=R\left(\tau^{-1} \sigma\right)=R\left(t^{-1} s\right)=R(s) .
$$

Since $R$ is multiplicative on $S$ and $R(\gamma \sigma)=R(r s)$, we also have $R(r)=R(\gamma)$. From the assumption $\Gamma_{0} \tau^{-1} m \sigma \Gamma_{0}=\Gamma_{0} t^{-1} n s \Gamma_{0}$ it follows at once that

$$
\Gamma_{0} \tau^{-1} \Gamma_{0} m \Gamma_{0} \sigma \Gamma_{0}=\Gamma_{0} t^{-1} \Gamma_{0} n \Gamma_{0} s \Gamma_{0},
$$

at which point it becomes convenient to let $x:=r t=\gamma \tau$ and $y:=r s=\gamma \sigma$, and to write $\tau, \sigma, t$ and $s$ in terms of $x, y, r$ and $\gamma$. Multiplication of (1.2) on the left by $x$ and on the right by $y^{-1}$ yields

$$
\left(x \Gamma_{0} x^{-1}\right)\left(\gamma \Gamma_{0} m \Gamma_{0} \gamma^{-1}\right)\left(y \Gamma_{0} y^{-1}\right)=\left(x \Gamma_{0} x^{-1}\right)\left(r \Gamma_{0} n \Gamma_{0} r^{-1}\right)\left(y \Gamma_{0} y^{-1}\right) .
$$

Using the multiplication rules in $\mathcal{H}\left(\Gamma, \Gamma_{0}\right)$ we see that

$\left(x \Gamma_{0} x^{-1}\right)$ is the support of $\mu_{x} \mu_{x}^{*}$,

$\left(y \Gamma_{0} y^{-1}\right)$ is the support of $\mu_{y} \mu_{y}^{*}$,

$\left(\gamma \Gamma_{0} m \Gamma_{0} \gamma^{-1}\right)$ is the support of $\mu_{\gamma}[m] \mu_{\gamma}^{*}$, and

$\left(r \Gamma_{0} n \Gamma_{0} r^{-1}\right)$ is the support of $\mu_{r}[n] \mu_{r}^{*}$.

It follows that

$$
\mu_{x} \mu_{x}^{*} \mu_{\gamma}[m] \mu_{\gamma}^{*} \mu_{y} \mu_{y}^{*}=\mu_{x} \mu_{x}^{*} \mu_{r}[n] \mu_{r}^{*} \mu_{y} \mu_{y}^{*},
$$

because the supports match and since $R(\gamma)=R(r)$ the coefficients also coincide. Now simply multiply by $\mu_{x}^{*}$ on the left, by $\mu_{y}$ on the right, and simplify $\mu_{x}^{*} \mu_{x} \mu_{x}^{*} \mu_{\gamma}=$ $\mu_{\tau}^{*}$ and other similar products to see that $\mu_{\tau}^{*}[m] \mu_{\sigma}=\mu_{t}^{*}[n] \mu_{s}$. This finishes the proof of the lemma and thus of part (i).

It is possible to give a direct computational proof of part (ii) along the lines of [9, 1, 5], but it is more efficient to use the Hecke algebra as in Remark 1.6. To this effect notice that, because of relation $\left(\mathfrak{h}_{3}\right)$ with $x \in \Gamma_{0}$, the projection $\mu_{s} \mu_{s}^{*}$ is contained in $\mathcal{H}\left(N, \Gamma_{0}\right)$ for every $s \in S$. Since $\mu_{s}$ is a semigroup of isometries by relation $\left(\mathfrak{h}_{1}\right)$, the left-hand side of $\left(\mathfrak{h}_{3}\right)$ defines a semigroup of injective corner 
endomorphisms: the image of $\alpha_{s}$ is the corner $\mu_{s} \mu_{s}^{*} \mathcal{H}\left(N, \Gamma_{0}\right) \mu_{s} \mu_{s}^{*}$, on which $\alpha_{s}$ has an inverse defined by $X \mapsto \mu_{s}^{*} X \mu_{s}$.

A standard reinterpretation of the relations $\left(\mathfrak{h}_{1}, \mathfrak{h}_{2}, \mathfrak{h}_{3}\right)$ shows that they are in fact a presentation of the ${ }^{*}$-algebraic semigroup crossed product $\mathcal{H}\left(N, \Gamma_{0}\right) \rtimes_{\alpha} S$. Since the $\mu_{s}$ 's and $e(x)$ 's in the Hecke algebra satisfy these relations, there is a canonical homomorphism of $\mathcal{H}\left(N, \Gamma_{0}\right) \rtimes_{\alpha} S$ to $\mathcal{H}\left(\Gamma, \Gamma_{0}\right)$, which we must show is an isomorphism. In order to avoid confusion we temporarily denote the generators of the universal *-algebra with the given presentation by $\tilde{\mu}_{s}$ and $\tilde{e}(x)$ while maintaining $\mu_{s}$ and $e(x)$ for the generators of the Hecke algebra. Thus, the canonical homomorphism maps $\tilde{\mu}_{t}^{*} \tilde{e}(n) \tilde{\mu}_{s}$ into $\mu_{t}^{*} e(n) \mu_{s}$, respectively, and we will show that it is an isomorphism by showing that this map is one-to-one from a spanning set onto the linear basis from part (i). Indeed, the linear span of products of the form $\tilde{\mu}_{t}^{*} \tilde{e}(x) \tilde{\mu}_{s}$ is multiplicatively closed, so it must be all of $\mathcal{H}\left(N, \Gamma_{0}\right) \rtimes_{\alpha} S$; see for example Remark 1.6 of [11. Suppose now that $\mu_{\tau}^{*} e(m) \mu_{\sigma}=\mu_{t}^{*} e(n) \mu_{s}$. Then (11.3) holds and, by $\left(\mathfrak{h}_{3}\right)$, it corresponds to a relation satisfied in the subalgebra $\mathcal{H}\left(N, \Gamma_{0}\right)$. But the elements $\tilde{e}(x)$ satisfy all the relations that are satisfied in $\mathcal{H}\left(N, \Gamma_{0}\right)$, because $\left(\mathfrak{h}_{2}\right)$ is a presentation of $\mathcal{H}\left(N, \Gamma_{0}\right)$, so it follows, again using $\left(\mathfrak{h}_{3}\right)$, that (1.3) also holds for the corresponding universal elements $\tilde{\mu}$ and $\tilde{e}$. On multiplying this universal version of (1.3) on the left by $\tilde{\mu}_{x}^{*}$ and on the right by $\tilde{\mu}_{y}$, and simplifying the result using $\left(\mathfrak{h}_{1}\right)$, as in the end of the proof of Lemma 1.10, we see that $\tilde{\mu}_{\tau}^{*} \tilde{e}(m) \tilde{\mu}_{\sigma}=\tilde{\mu}_{t}^{*} \tilde{e}(n) \tilde{\mu}_{s}$. This finishes the proof of (ii) and of the theorem.

An immediate application of Theorem 1.9 and the corresponding universal properties gives the following result about $\mathrm{C}^{*}$-algebras.

Theorem 1.11. Under the assumptions of the preceding theorem, the universal Hecke $C^{*}$-algebra $C_{u}^{*}\left(\Gamma, \Gamma_{0}\right)$ exists if and only if $C_{u}^{*}\left(N, \Gamma_{0}\right)$ exists, in which case the isomorphisms of part (ii) extend to $C^{*}$-algebra isomorphisms:

$$
C_{u}^{*}\left(\Gamma, \Gamma_{0}\right) \cong C_{u}^{*}\left(N, \Gamma_{0}\right) \rtimes_{\alpha} S \cong C_{u}^{*}\left(\mathfrak{h}_{1}, \mathfrak{h}_{2}, \mathfrak{h}_{3}\right) .
$$

Notice that, by [11] Theorem 2.1.1], the first isomorphism implies that $C_{u}^{*}\left(N, \Gamma_{0}\right)$ embeds in $C_{u}^{*}\left(\Gamma, \Gamma_{0}\right)$.

Corollary 1.12. If $\Gamma_{0}$ is normal in $N$, then relation $\left(\mathfrak{h}_{2}\right)$ defines the group algebra of $N / \Gamma_{0}$ so one has

$$
\mathcal{H}\left(\Gamma, \Gamma_{0}\right) \cong \mathbb{C}\left(N / \Gamma_{0}\right) \rtimes_{\alpha} S \quad \text { and } \quad C_{u}^{*}\left(\Gamma, \Gamma_{0}\right) \cong C^{*}\left(N / \Gamma_{0}\right) \rtimes_{\alpha} S .
$$

Remark 1.13. By the dilation/extension theorems of [11] the representation theory of $\mathcal{H}\left(\Gamma, \Gamma_{0}\right)$ is equivalent to that of a $\mathrm{C}^{*}$-dynamical system $(A, G, \beta)$ in which $A$ is the inductive $\operatorname{limit}_{\lim _{S}}\left(\mathcal{H}\left(N, \Gamma_{0}\right), \alpha_{s}\right)$ and $\beta$ is an action of $G$ by automorphisms of $A$ (we refer to the appendix of [14] for the categorical statement of this equivalence). This is particularly useful when $\left(N, \Gamma_{0}\right)$ is a Gelfand pair, that is to say, when $\mathcal{H}\left(N, \Gamma_{0}\right)$ is commutative, since it then allows one to apply results about transformation group $\mathrm{C}^{*}$-algebras to Hecke $\mathrm{C}^{*}$-algebras. See for instance [10] and 14]. 


\section{EXAMPLES}

Example 2.1. Theorem 1.9 applies to the Bost-Connes Hecke $\mathrm{C}^{*}$-algebra from [4] and to the Hecke $\mathrm{C}^{*}$-algebras of the inclusions

$$
\left(\begin{array}{ll}
1 & \mathcal{O} \\
0 & 1
\end{array}\right) \subset\left(\begin{array}{cc}
1 & \mathcal{K} \\
0 & \mathcal{K}^{*}
\end{array}\right)
$$

where $\mathcal{K}$ is a number field with ring of integers $\mathcal{O}$. These have already been shown to be semigroup crossed products in [9] and [1], and also in [5] under the additional assumption that $\mathcal{K}$ is of class number 1 . For the case when $\mathcal{O}$ is one of the principal subrings of a global field $\mathcal{K}$, see 13 .

Example 2.2. Now we briefly recall the situation discussed in Example 4.3 of [5]. Let $G=G L_{d}^{+}(\mathbb{Q})$ be the group of rational $d \times d$ matrices with positive determinant, and let $S=G \cap M_{d}(\mathbb{Z})$ be the subsemigroup of matrices having integer entries. Then $G$ acts on $N=\mathbb{Q}^{d}$ by $\psi_{g}(n)=\left(g^{t}\right)^{-1} n$, where $g^{t}$ indicates the transpose of $g$ (we choose this action instead of the conventional action used in [5] in order to avoid having to switch from $S$ to $S^{-1}$ later on). Let $\Gamma:=N \rtimes G=\mathbb{Q}^{d} \rtimes G L_{d}^{+}(\mathbb{Q})$, and consider the subgroup $\Gamma_{0}=\mathbb{Z}^{d} \subset \mathbb{Q}^{d}=N$. It is clear that if $s \in S$, then $\psi_{s^{-1}}\left(\Gamma_{0}\right)=s^{t} \mathbb{Z}^{d} \subset \mathbb{Z}^{d}$ and since $\left|\Gamma_{0} / \psi_{s^{-1}}\left(\Gamma_{0}\right)\right|=\operatorname{det}\left(s^{t}\right)<\infty$, we have a finite order inclusion for every $s \in S$.

From Theorem 1.9 we deduce that $\left(\mathbb{Q}^{d} \rtimes G L_{d}^{+}(\mathbb{Q}), \mathbb{Z}^{d}\right)$ is a Hecke pair and that its Hecke $\mathrm{C}^{*}$-algebra is isomorphic to the semigroup crossed product

$$
C_{u}^{*}\left(\mathbb{Q}^{d} \rtimes G L_{d}^{+}(\mathbb{Q}), \mathbb{Z}^{d}\right) \cong C^{*}\left(\mathbb{Q}^{d} / \mathbb{Z}^{d}\right) \rtimes_{\alpha} S .
$$

Remark 2.3. Theorem 2.11 of [5] suffices for the existence of this universal Hecke $\mathrm{C}^{*}$-algebra, but it does not give the semigroup crossed product structure, because in this case the normalizer of $\Gamma_{0}$ is not normal in $\Gamma$.

Next we shall use a multivariable version of the arguments of Section 3 of [1] to obtain an explicit realization of the minimal dilated system whose crossed product contains the Hecke $\mathrm{C}^{*}$-algebra as a full corner. Denote by $\mathcal{A}_{f}$ the ring of finite adeles over $\mathbb{Q}$, with $\mathcal{R}=\prod_{p} \mathbb{Z}_{p}$ the maximal compact subring. Corresponding to the natural action $(g, x) \mapsto g x$ of $G$ on $\mathcal{A}_{f}^{d}$ there is an action $\beta$ of $G$ on $C_{0}\left(\mathcal{A}_{f}^{d}\right)$ given by $\left(\beta_{g} F\right)(x)=F\left(g^{-1} x\right)$ for $F \in C_{0}\left(\mathcal{A}_{f}^{d}\right)$.

Proposition 2.4. The Hecke $C^{*}$-algebra $C_{u}^{*}\left(\mathbb{Q}^{d} \rtimes G L_{d}^{+}(\mathbb{Q}), \mathbb{Z}^{d}\right)$ is (isomorphic to) the full corner in the crossed product $C_{0}\left(\mathcal{A}_{f}^{d}\right) \rtimes_{\beta} G L_{d}^{+}(\mathbb{Q})$ corresponding to the projection $1_{\mathcal{R}^{d}} \in \mathcal{A}_{f}^{d}$.

Proof. Observe first that the duality pairing of $\mathbb{Q}^{d} / \mathbb{Z}^{d}$ with $\mathcal{R}^{d}$ given by

$$
\langle q, x\rangle=\exp \left(2 \pi i \sum_{j=1}^{d} q_{j} x_{j}\right)
$$

establishes a Gelfand-Fourier transform isomorphism of $C^{*}\left(\mathbb{Q}^{d} / \mathbb{Z}^{d}\right)$ to $C\left(\mathcal{R}^{d}\right)$. To see that the action $\beta$ restricted to $S$ is conjugate to the semigroup action of $S$ on $C^{*}\left(\mathbb{Q}^{d} / \mathbb{Z}^{d}\right)$ from part (ii) of Theorem 1.9 it is enough to carry out the computation on the characters $e(x)=\langle x, \cdot\rangle$. Thus

$$
\alpha_{s}(e(x))=R(s)^{-1} \sum_{\left[s^{t} y=x\right]} e(y)
$$


yields

$$
\hat{\alpha}_{s}(\langle x, \cdot\rangle)=R(s)^{-1} \sum_{\left[s^{t} y=x\right]}\langle y, \cdot\rangle=R(s)^{-1} \sum_{\left[s^{t} y=x\right]}\left\langle\left(s^{t}\right)^{-1} s^{t} y, \cdot\right\rangle
$$

which, when evaluated at $w \in \mathcal{R}^{d}$, equals $\left\langle x, s^{-1} w\right\rangle$ or zero, according to whether $w \in s \mathcal{R}^{d}$ or not. Hence $\beta_{s} \circ i=i \circ \alpha_{s}$, where $i: C\left(\mathcal{R}^{d}\right) \rightarrow C_{0}\left(\mathcal{A}_{f}^{d}\right)$ is the obvious inclusion.

Since for every $s \in S$ there is a positive integer $m \in \mathbb{N}^{\times}$such that $m s^{-1} \in S$, the semigroup $\mathbb{N}^{\times}$of multiplicative positive integers is cofinal in $S$, and from this it follows easily that $\bigcup_{S} \beta_{s^{-1}} C\left(\mathcal{R}^{d}\right)$ is dense in $C_{0}\left(\mathcal{A}_{f}^{d}\right)$, because $\bigcup_{m \in \mathbb{N}^{\times}} m^{-1} \mathcal{R}^{d}$ is dense in $\mathcal{A}_{f}^{d}$. The result now follows from Theorems 2.1 and 2.4 of [11.

Example 2.5. Suppose $\mathcal{K}$ is a number field with ring of integers $\mathcal{O}$, and fix an integer $d \geq 1$. Define

$$
\begin{aligned}
G & :=G L_{d}(\mathcal{K}), \\
S & :=G L_{d}(\mathcal{K}) \cap \mathcal{M}_{d}(\mathcal{O}), \\
\Gamma_{0} & :=\mathcal{O}^{d}, \\
N & :=\mathcal{K}^{d},
\end{aligned}
$$

and let $\Gamma:=N \rtimes G$ where the action of $g \in G$ on $N$ is by $\psi_{g}(n)=\left(g^{t}\right)^{-1} n$.

Proposition 2.6. With the above notation, $\left(\Gamma, \Gamma_{0}\right)$ is a Hecke pair, whose Hecke $C^{*}$-algebra is isomorphic to the semigroup crossed product $C^{*}\left(\mathcal{K}^{d} / \mathcal{O}^{d}\right) \rtimes_{\alpha} S$, and to a corner, corresponding to the characteristic function of the maximal compact subring $\mathcal{R}_{\mathcal{K}}^{d}$ of $\mathcal{A}_{\mathcal{K}}^{d}$, in the crossed product $C_{0}\left(\mathcal{A}_{\mathcal{K}}^{d}\right) \rtimes_{\beta} G L_{d}(\mathcal{K})$, where $\beta$ denotes the action of $G L_{d}(\mathcal{K})$ on $C_{0}\left(\mathcal{A}_{\mathcal{K}}^{d}\right)$ given by $\beta_{g}(f)(x)=f\left(g^{-1} x\right)$.

The proof consists of an application of Theorem 1.9 and of Theorems 2.1 and 2.4 of [11], and is entirely analogous to that of the preceding example.

Example 2.7. Motivated by Example 4.6 of [5] we consider the following situation. Let $R$ be a commutative unital ring and let $\Gamma_{0}$ be an $R$-module. Suppose $S \subset R$ is a multiplicatively closed set without zero-divisors, so that one has localizations $S^{-1} R$ of the ring $R$ and $S^{-1} \Gamma_{0}$ of the module $\Gamma_{0}$ that contain, respectively, copies of $R$ and $\Gamma_{0}$.

Then $G=S^{-1} S$ is a subgroup of the unit group of $S^{-1} R$, there is an action $\psi$ of $G$ on $N:=S^{-1} \Gamma_{0}$ given simply by $\psi_{g}(n)=g^{-1} n$, and we define $\Gamma:=N \rtimes G$.

Proposition 2.8. Assume $\left|s^{-1} \Gamma_{0} / \Gamma_{0}\right|<\infty$ for each $s \in S$ and let $\Gamma:=S^{-1} \Gamma_{0} \rtimes$ $S^{-1} S$. Then $\left(\Gamma, \Gamma_{0}\right)$ is a Hecke pair and its Hecke algebra (respectively $C^{*}$-algebra) is the semigroup crossed product $\mathbb{C}\left(S^{-1} \Gamma_{0} / \Gamma_{0}\right) \rtimes S$ ) (respectively $C^{*}\left(S^{-1} \Gamma_{0} / \Gamma_{0}\right) \rtimes$ $S)$.

Example 2.9. In the above examples $\Gamma_{0}$ is normal in $N$, so Corollary 1.12 is all that is needed. Next we borrow from [12] an interesting example arising in number theory in which the inclusion $\Gamma_{0} \subset N$ is not normal, so that the full generality of Theorems 1.9 and 1.11 is needed. Let $\mathcal{K}$ be a number field, with ring of integers $\mathcal{O}$, and assume further that $\mathcal{K}$ is quadratic imaginary, so that the unit group $\mathcal{O}^{*}$ is finite. Consider the group inclusion $\Gamma_{0} \subset \Gamma$ with $\Gamma_{0}:=\left(\begin{array}{cc}1 & \mathcal{O} \\ 0 & \mathcal{O}^{*}\end{array}\right)$ and $\Gamma:=\left(\begin{array}{cc}1 & \mathcal{K} \\ 0 & \mathcal{K}^{*}\end{array}\right)$. The map $\left(\begin{array}{cc}1 & y \\ 0 & x\end{array}\right) \mapsto x \mathcal{O}^{*}$ is a homomorphism from $\Gamma$ onto the group $G:=\mathcal{K}^{*} / \mathcal{O}^{*}$ 
of principal (fractional) ideals of $\mathcal{K}$; its kernel is $N:=\left(\begin{array}{cc}1 & \mathcal{K} \\ 0 & \mathcal{O}^{*}\end{array}\right)$. In general $\Gamma_{0}$ is not normal in $N$, but the double $\Gamma_{0}$-coset of $\left(\begin{array}{cc}1 & y \\ 0 & x\end{array}\right) \in N$ is $\left(\begin{array}{cc}1 & y \mathcal{O}^{*}+\mathcal{O} \\ 0 & \mathcal{O}^{*}\end{array}\right)$, which contains at most $\left|\mathcal{O}^{*}\right|$ right cosets, so $\left(N, \Gamma_{0}\right)$ is a Hecke pair.

Let $S:=\mathcal{O}^{\times} / \mathcal{O}^{*}$ be the semigroup of principal integral ideals, and let $\sigma$ : $\left(\mathcal{O}^{\times} / \mathcal{O}^{*}\right) \rightarrow \mathcal{O}^{\times}$be a cross section of $\mathcal{O}^{\times} \rightarrow\left(\mathcal{O}^{\times} / \mathcal{O}^{*}\right)$ (see for example [12, Lemma $2.5])$. Then $\left(a \mathcal{O}^{*}\right) \mapsto\left(\begin{array}{cc}1 & 0 \\ 0 & \sigma\left(a \mathcal{O}^{*}\right)\end{array}\right)$ determines a cross section $\tilde{\sigma}$ of the quotient $\Gamma \rightarrow \mathcal{K}^{*} / \mathcal{O}^{*}$, so we have that $\Gamma$ is a semidirect product

$$
\left(\begin{array}{cc}
1 & \mathcal{K} \\
0 & \mathcal{O}^{*}
\end{array}\right) \rtimes\left(\mathcal{K}^{*} / \mathcal{O}^{*}\right)
$$

in which the action of $\mathcal{K}^{*} / \mathcal{O}^{*}$ is given by $\psi_{g}\left(\begin{array}{cc}1 & y \\ 0 & u\end{array}\right):=\tilde{\sigma}(g)\left(\begin{array}{cc}1 & y \\ 0 & u\end{array}\right) \tilde{\sigma}(g)^{-1}$.

For each $a \mathcal{O}^{*} \in S$ the subgroup $\tilde{\sigma}(a)^{-1} \Gamma_{0} \tilde{\sigma}(a)=\left(\begin{array}{cc}1 & a \mathcal{O} \\ 0 & \mathcal{O}^{*}\end{array}\right)$ is of finite order in $\Gamma_{0}$ because $|\mathcal{O} / a \mathcal{O}|<\infty$. It follows from Theorem 1.9 that the pair $\left(\mathcal{K} \rtimes \mathcal{K}^{*}, \mathcal{O} \rtimes \mathcal{O}^{*}\right)$ is a Hecke pair and that

$$
\mathcal{H}\left(\mathcal{K} \rtimes \mathcal{K}^{*}, \mathcal{O} \rtimes \mathcal{O}^{*}\right) \cong \mathcal{H}\left(\mathcal{K} \rtimes \mathcal{O}^{*}, \mathcal{O} \rtimes \mathcal{O}^{*}\right) \rtimes\left(\mathcal{O}^{\times} / \mathcal{O}^{*}\right) .
$$

Moreover, the pair $\left(\mathcal{K} \rtimes \mathcal{O}^{*}, \mathcal{O} \rtimes \mathcal{O}^{*}\right)$ has a universal Hecke $\mathrm{C}^{*}$-algebra, and $C_{u}^{*}\left(\Gamma, \Gamma_{0}\right)$ $\cong C_{u}^{*}\left(N, \Gamma_{0}\right) \rtimes\left(\mathcal{O}^{\times} / \mathcal{O}^{*}\right)$.

Remark 2.10. The assumption that $\mathcal{K}$ be quadratic imaginary is not necessary for the result, but simplifies the argument; we refer to [12] for the proof of the general statement. Here we limit ourselves to point out that $C_{u}^{*}\left(N, \Gamma_{0}\right)$ exists because its generators have finite spectrum and hence uniformly bounded norm in any representation, and that the action of the principal integral ideals $\mathcal{O}^{\times} / \mathcal{O}^{*}$ by endomorphisms of $\mathcal{H}\left(\mathcal{K} \rtimes \mathcal{O}^{*}, \mathcal{O} \rtimes \mathcal{O}^{*}\right)$ is independent of the cross section $\sigma$, because the subgroup $\left(\begin{array}{cc}1 & 0 \\ 0 & \mathcal{O}^{*}\end{array}\right)$ leaves $\Gamma_{0}$ invariant.

\section{REFERENCES}

[1] J. Arledge, M. Laca and I. Raeburn, Semigroup crossed products and Hecke algebras arising from number fields, Documenta Math. 2 (1997), 115-138. MR 98k:46111

[2] M. W. Binder, Induced factor representations of discrete groups and their types, J. Funct. Anal. 115 (1993), 294-312. MR 94g:22010

[3] B. Blackadar, Shape theory for $C^{*}$-algebras, Math. Scand. 56 (1985), 249-275. MR 87b:46074

[4] J.-B. Bost and A. Connes, Hecke algebras, Type III factors and phase transitions with spontaneous symmetry breaking in number theory, Selecta Math. (New Series) 1 (1995), 411-457. MR 96m:46112

[5] B. Brenken, Hecke algebras and semigroup crossed product $C^{*}$-algebras, Pacific J. Math. 187 (1999), 241-262. MR 2000g:46071

[6] R. W. Hall, Hecke $C^{*}$-algebras, Ph.D. thesis, The Pennsylvania State University, December 1999.

[7] A. Krieg, Hecke Algebras, Mem. Amer. Math. Soc. 87 (1990), No. 435. MR 90m:16024

[8] M. Laca and I. Raeburn, Semigroup crossed products and the Toeplitz algebras of nonabelian groups, J. Funct. Anal. 139 (1996), 415-440. MR 97h:46109

[9] M. Laca and I. Raeburn, A semigroup crossed product arising in number theory, J. London Math. Soc. 59 (1999), 330-344. MR 2000g:46097

[10] M. Laca and I. Raeburn, The ideal structure of the Hecke $C^{*}$-algebra of Bost and Connes, Math. Ann. 318 (2000), 433-451. MR 2002a:46095

[11] M. Laca, From endomorphisms to automorphisms and back: dilations and full corners, J. London Math. Soc. (2) 61 (2000), 893-904. MR 2002a:46094 
[12] M. Laca, M. van Frankenhuysen, Phase transitions on Hecke $C^{*}$-algebras and class field theory, in preparation.

[13] N. S. Larsen and I. Raeburn, Faithful representations of crossed products by actions of $\mathbb{N}^{k}$, Math. Scand. 89 (2001), 283-296.

[14] N. S. Larsen and I. Raeburn, Representations of Hecke algebras and dilations of semigroup crossed products, J. London Math. Soc., to appear.

[15] G. J. Murphy, Crossed products of $C^{*}$-algebras by endomorphisms, Integral Equations \& Operator Theory 24 (1996), 298-319. MR 97f:46105

[16] K. Tzanev, C*-algèbres de Hecke et K-theorie, Thèse de Doctorat, Université de Paris 7 , December 2000.

Department of Mathematics, University of Münster, 48149 Münster, Germany

E-mail address: laca@math.uni-muenster.de

Current address: Department of Mathematics and Statistics, University of Victoria, Victoria, British Columbia, Canada V8W 3P4

Department of Mathematics, University of Copenhagen, Universitetsparken 5, DK2100 Copenhagen $\varnothing$, Denmark

E-mail address: nadia@math.ku.dk 